\title{
A Pedestrian Navigation System Based on Low Cost IMU
}

\author{
Yan Li and Jianguo Jack Wang \\ (University of Technology, Sydney, Australia) \\ (E-mail: jianguo.wang@uts.edu.au)
}

\begin{abstract}
For indoor pedestrian navigation with a shoe-mounted inertial measurement unit (IMU), the zero velocity update (ZUPT) technique is implemented to constrain the sensors' error. ZUPT uses the fact that a stance phase appears in each step at zero velocity to correct IMU errors periodically. This paper introduces three main contributions we have achieved based on ZUPT. Since correct stance phase detection is critical for the success of applying ZUPT, we have developed a new approach to detect the stance phase of different gait styles, including walking, running and stair climbing. As the extension of ZUPT, we have proposed a new concept called constant velocity update (CUPT) to correct IMU errors on a moving platform with constant velocity, such as elevators or escalators where ZUPT is infeasible. A closedloop step-wise smoothing algorithm has also been developed to eliminate discontinuities in the trajectory caused by sharp corrections. Experimental results demonstrate the effectiveness of the proposed algorithms.
\end{abstract}

\author{
KEYWORDS \\ 1. Pedestrian Navigation. 2. Inertial Measurement Unit (IMU). \\ 3. Zero Velocity Update (ZUPT). 4. Constant Velocity Update (CUPT).
}

Submitted: 26 August 2013. Accepted: 11 May 2014.

1. INTRODUCTION. Obtaining seamless navigation in all circumstances, including an environment with degraded Global Positioning System (GPS) signals, is a challenging issue. An Inertial Navigation System (INS) can provide continuous navigation information independently but accumulates errors, which need to be corrected frequently by additional measurements. GPS/INS integration has received much attention in navigation applications. Godha and Lachapelle (2008) proposed a system that combined a shoe-mounted Inertial Measurement Unit (IMU) and GPS to bound drift errors in outdoor scenarios.

For pedestrian navigation in an indoor environment where the GPS signal is unreliable, some non-GPS approaches require external references for tracking and navigation. Hide et al. (2010) investigated the use of computer vision-derived velocity measurements to frequently correct the drift of a low-cost IMU. Aoki et al. (1999) proposed an image sequence matching technique for the recognition of locations and previously visited places. Work is also being done on simultaneous location and 
mapping (SLAM), which usually uses cameras as sensors. However, unlike the inertial sensor, these sensors are very sensitive to the environment and are unreliable in unfavourable operating conditions.

Compared to other types of navigation technologies, the main advantages of inertial sensor-based systems are that they are self-contained, environmentindependent and can provide continuous navigation information. These meet the requirements of robust pedestrian navigation, not requiring an instrumented, marked, or pre-mapped environment. Meanwhile, the major challenge for IMU navigation alone is how to efficiently reduce the integral drift which is the primary cause of errors in inertial navigation. Whether a pedestrian is walking, running or climbing stairs, there is a stance phase in each step that can provide a zero velocity measurement for error correction of a shoe-mounted IMU. This approach is called zero velocity update (ZUPT). Consequently it is critical to correctly identify the stance phase in each step and then to apply ZUPT for IMU error correction.

Numerous ZUPT-based pedestrian navigation systems have been developed using various approaches for stance phase detection. Foxlin (2005) developed a system called NavShoe to use the stance phase of walking as measurements into an extended Kalman filter (EKF) for performing ZUPT. Castaneda and Lamy-Perbal (2010) proposed an improved shoe-mounted inertial navigation system based on the fuzzy logic step detector and an angular updating algorithm. Cho and Park (2006) proposed a pedometer-like approach which counts steps and estimates average length of steps. Step length is estimated from accelerometer readings that are passed through a neural network, and a Kalman filter was used to reduce the effect of magnetic disturbances.

Alternatively, a dead reckoning method of propagating the step length over a certain direction is often used. Huang et al. (2010) proposed a method that fuses both strap-down inertial navigation and pedestrian dead-reckoning methods. Feliz et al. (2009) proposed using a compass and gyroscope to determine trajectories and correct sensor measurements, along with an integrated barometer for height measurement. A similar zero-velocity detector constructed around a pressure sensor mounted beneath the heel of a shoe was used with good results (Bebek et al., 2010). Jiménez et al. (2009) implemented and compared several of the most relevant algorithms for step detection, stride length, heading and position estimation. Tests were conducted and it was found that the stride length estimation errors were only about $1 \%$. The positioning errors were almost always below $5 \%$ of the total travelled distance. The main source of positioning errors was orientation estimation.

The algorithms for step detection using accelerometers mainly consist of three types: peak detection, zero crossing detection and flat zone detection (Cho et al., 2003). A gait state is modelled as a Markov process and gait states are estimated using the hidden Markov model filter based on force sensors to determine when to apply ZUPT (Suh and Park, 2009). Similarly, a Markov model is constructed using segmentation of gyroscope outputs (Park and Suh, 2010). Different step detection algorithms are developed based on both accelerometer and gyroscope outputs. The zero velocity is determined by comparing z-axis accelerometer and y-axis gyroscope outputs with proper threshold values (Yun et al., 2007). Alternatively, the zero velocity is determined based on norms of accelerometer and gyroscope along with variance of the accelerometer. Then a Kalman filter-based framework, called INSEKF-ZUPT, is implemented (Jiménez et al., 2010). Skog et al. (2010) developed a novel stance hypothesis optimal detector; however this is restricted to $2 \mathrm{D}$ cases. 
Ojeda and Borenstein (2007) implemented two empiric rules for gyro signals analysis to detect zero velocity. The system was tested with a variety of walking patterns, on stairs and on rugged terrain. The horizontal relative error is about $0.49 \%$ of the total distance travelled; the vertical error is $1.2 \%$, which might be the highest accuracy in related work.

However, all the step detection algorithms mentioned above only function for walking, and fail to detect stance phases in high-speed movement (running or jumping). Compared with walking, the duration of the stance phase in running is shorter and the velocity in the stance phase is less close to zero velocity. Barely enlarging the threshold will introduce false stance phase detection. It is a challenge to correctly detect stance phases in all the moving styles.

Moreover, none of the shoe-mounted MEMS inertial sensors-based pedestrian navigation systems, using strap-down inertial navigation or pedestrian dead-reckoning methods, can perform well in situations with constant velocity, such as in an elevator or on an escalator. This is due to the fact that they treat stance phases always as zero velocity even if a sensor is not stationary but moving with a constant velocity.

As the errors of estimated position and velocity grow rapidly with time for shoe-mounted MEMS inertial sensors, ZUPT applied at each step leads to sharp corrections of velocity and position; the estimated trajectories have discontinuities and periodic drift, which is unpleasant in accurate navigation and tracking. Colomar and his group (Colomar et al., 2012) developed an open loop step-wise smoothing algorithm to aid ZUPT that can efficiently eliminate the sharp corrections and discontinuities at the end of the step.

This paper describes our contributions on shoe-mounted inertial sensor navigation on three aspects. We have proposed a robust stance phase detection algorithm to handle both walking and running ( $\mathrm{Li}$ and Wang, 2012). The new algorithm consists of a footstep detector to indicate a new step and a swing phase detector to inform the end of the step, a stance phase detector is applied to detect whether the foot is stationary. As the extension of ZUPT, a new concept called CUPT has been proposed to apply velocity correction when a pedestrian is on an escalator or in an elevator ( $\mathrm{Li}$ et al., 2012). CUPT can broaden the practical use of a shoe-mounted pedestrian navigation system in a building environment. As an improvement of Colomar's open loop stepwise smoothing, a closed-loop step-wise smoothing algorithm is implemented to eliminate the sharp corrections over the steps (Li and Wang, 2013).

This paper is organised as follows. Section 2 briefly introduces the general background of a standard strap-down INS mechanism; the framework of ZUPTaided INS and EKF error model applied in pedestrian navigation. Section 3 presents the stance phase detection for different gait styles, including walking, running and stair climbing. The new concept CUPT for correcting IMU errors on a moving platform with constant velocity is introduced in Section 4 and a closed-loop step-wise smoothing algorithm is introduced in Section 5. Experimental results and discussions are presented in Section 6 and the paper is concluded in Section 7.

2. INERTIAL PEDESTRIAN NAVIGATION. The proposed shoemounted inertial navigation system uses only a MEMS IMU, which consists of accelerometers, gyroscopes and magnetometers. The system adopts the standard strap-down inertial navigation mechanization, with zero/constant velocity as the 


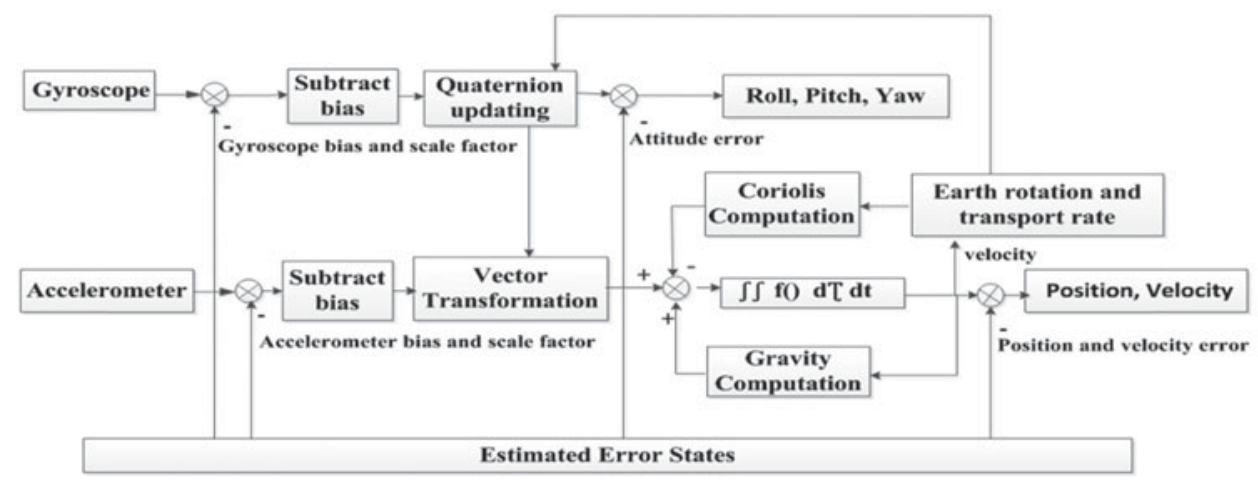

Figure 1. The basic blocks of strap-down inertial navigation mechanization.

measurement for correction. Figure 1 shows the system mechanization. Attitude can be determined by integration of the rotation rate of gyros. After vector transformation, velocity and position can be determined by the integration of acceleration with compensation of gravity and Coriolis force. If no estimated error states (EES) provide correcting information however, due to the bias of the sensors and the noise associated with the measurement, this simple integration in practice leads to unbounded growth in position errors with time.

2.1. ZUPT/CUPT. In order to obtain a high accuracy navigation solution with a shoe-mounted IMU, ZUPT/CUPT can be applied to reduce the errors. They use the fact that when a pedestrian travels, one's feet alternate between a stance phase and a swing phase. If the stance phase appearing in each step can be detected correctly at a known (zero or constant) velocity, the INS velocity errors can be corrected periodically. More detailed explanation about ZUPT/CUPT can be found in Foxlin (2005) and Li et al. (2012).

It is important to correctly identify the stance phases of the IMU attached to a shoe and then apply ZUPT/CUPT to bind the navigation errors by providing proper EES (see Figure 1) with EKF or other estimation algorithms. There are two types of error in stance phase detection: misdetection and false-detection. It is noticed that a stance phase misdetection causes less serious problems for navigation accuracy than a false-detection. A misdetection just misses a chance for INS correction while a falsedetection results in a wrong 'correction' and may cause the navigation results to become useless.

In order to get a high accuracy navigation solution, ZUPT/CUPT is applied with an EKF to reduce navigation errors. The framework of the proposed navigation system is illustrated in Figure 2. It has three main blocks: 1) INS Mechanization to compute the position, velocity and attitude; 2) Stance Phase Detection block to determine stance phases and apply ZUPT/ CUPT; 3) EKF block to estimate the error states and bound navigation errors.

2.2. EKF. The EKF we used consists of 24 error states including navigation errors, sensor errors and gravity uncertainty. In each estimation cycle, once zero velocity has been detected, ZUPT delivers the updated error states to INS, the navigation error states are reset to zero after the current position, velocity and attitude is corrected. Other error states are accumulated and fed back to the inertial navigation mechanization. 


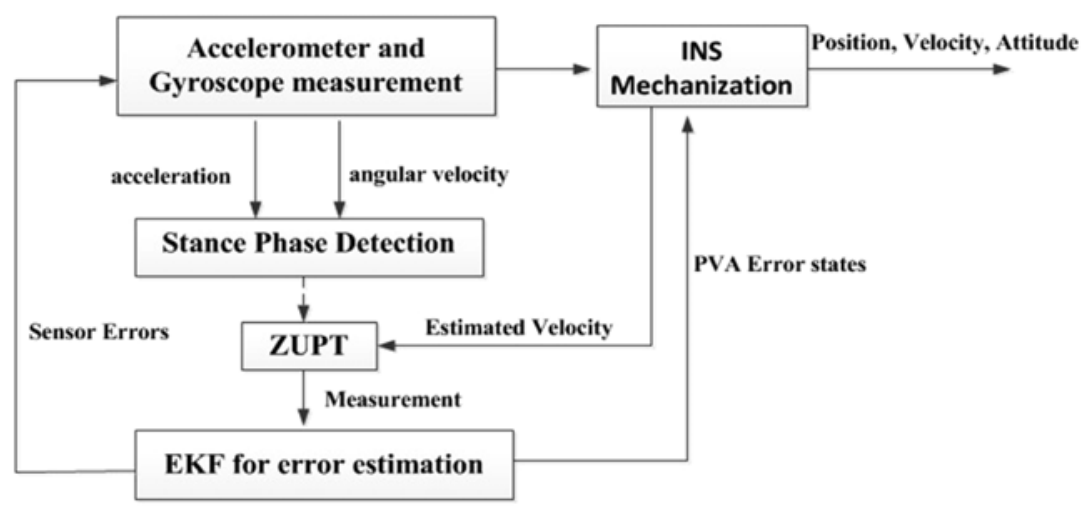

Figure 2. The main blocks in the framework used for pedestrian inertial navigation.

The EKF includes the following 24 error states:

$$
\begin{aligned}
\delta x_{N a v} & =\left[\delta r_{N}, \delta r_{E} \delta r_{D}, \delta v_{N} \delta v_{E}, \delta v_{D}, \delta \varphi_{H}, \delta \varphi_{P}, \delta \varphi_{R}\right]^{T} \\
\delta x_{I N S} & =\left[\nabla_{b}, \nabla_{f}, \varepsilon_{b}, \varepsilon_{f}\right] \\
\delta x_{\text {Grav }} & =\left[\delta g_{N}, \delta g_{E}, \delta g_{D}\right]
\end{aligned}
$$

where $\delta x_{N a v} \delta x_{I N S}$ and $\delta x_{\text {Grav }}$ are the navigation error vector, the IMU sensor measurement error vector and gravity uncertainty respectively. $\delta r \delta v$ and $\delta \varphi$ are the position, velocity and attitude error vectors. $\nabla$ expresses the accelerometer error vector, and $\varepsilon$ is the gyro error vector. Subscript $b$ stands for bias and subscript $f$ stands for scale factor.

The Psi-angle INS error model is adopted in the system (Grejner-Brzezinska et al., 2001):

$$
\begin{aligned}
& \delta \dot{r}=-w_{\text {en }} \times \delta r+\delta v \\
& \delta \dot{v}=-\left(w_{i e}+w_{\text {in }}\right) \times \delta v-\delta \varphi \times f+\delta g+\nabla \\
& \delta \dot{\varphi}=-w_{\text {in }} \times \delta \varphi+\varepsilon
\end{aligned}
$$

where $\delta \mathrm{r} \delta \mathrm{v} \delta \varphi$ are the position, velocity, and attitude error vectors respectively, $\nabla$ is the accelerometer error vector, $\delta \mathrm{g}$ is the error in the computed gravity vector, $\varepsilon$ is the gyro error vector, $f$ is the specific force vector, $w_{i e}$ is the earth rotation rate vector, $w_{e n}$ is the transport rate vector and $w_{i n}$ is the angular rate vector from the navigation to the inertial frame. The dynamic matrix is obtained by a linearization of Equation (2).

$$
\left[\begin{array}{c}
\delta \dot{x}_{\text {Nav }}^{1 \times 9} \\
\delta \dot{x}_{\text {grav }}^{1 \times 3} \\
\delta \dot{x}_{I M U}^{1 \times 12}
\end{array}\right]=\left[\begin{array}{ccc}
F_{11}^{9 \times 9} & F_{12}^{3 \times 9} & F_{13}^{12 \times 9} \\
0^{9 \times 3} & F_{22}^{3 \times 3} & 0^{12 \times 3} \\
0^{9 \times 12} & 0^{3 \times 12} & 0^{12 \times 12}
\end{array}\right]=\left[\begin{array}{c}
\delta x_{\text {Nav }} \\
\delta x_{\text {grav }} \\
\delta x_{I M U}
\end{array}\right]+\left[\begin{array}{c}
w_{\text {Nav }} \\
w_{\text {grav }} \\
w_{I M U}
\end{array}\right]
$$

where $w_{N a v} w_{I M U}$ and $w_{\text {grav }}$ are all zero-mean Gaussian white noise vectors. A detailed expression of the dynamic matrix can be found in Grejner-Brzezinska et al. (2001). 
For ZUPT/CUPT, the observation $z^{1 \times 3}$ is the velocity vector in three directions, and the measurement model is:

$$
z=H \delta x+\mathrm{n}
$$

where $\mathrm{H}^{3 \times 24}=\left[O^{3 \times 3} \mathrm{I}^{3 \times 3} \mathrm{O}^{3 \times 3}\right]$ and $\mathrm{n}$ is the measurement noise vector.

Consider the error state $\delta x=\hat{x}-x$ where $\hat{x}$ is the estimated state and $x$ is the true state, the observation $\mathrm{z}$ is $\hat{x}-0$ for ZUPT and $\hat{x}-v_{c}$ or CUPT, where $\hat{x}$ is computed by the strap-down inertial navigation mechanization and $v_{c}$ is the constant velocity.

In addition to pursuing reliable observation to improve an EKF's estimation, as we are doing here, some other approaches are also conducted, such as the quantization and coloured noises error modelling for inertial sensors (Han and Wang, 2011), and adaptive EKF (Wang et al., 2007). This paper's scope only covers the approaches for obtaining reliable observation with ZUPT/CUPT.

2.3. Stance Phase Detection. The stance phase detector we proposed consists of the following four logical conditions $\mathrm{C}_{1}-\mathrm{C}_{4}$, using both the accelerometer and gyro measurements. These four conditions must be satisfied $(=1)$ simultaneously to declare a foot as stationary for every sample epoch $\mathrm{k}$.

1) The magnitude of the acceleration $\left|a_{k}\right|$ where $a_{x k} a_{y k} a_{z k}$ are the magnitude of the acceleration on $\mathrm{x}, \mathrm{y}, \mathrm{z}$-axis respectively:

$$
\begin{aligned}
& \left|a_{k}\right|=\left[a_{x k}^{2}+a_{y k}^{2}+a_{z k}^{2}\right]^{1 / 2} \\
& C 1= \begin{cases}1 & \text { threshold }_{\text {amin }}<\left|a_{k}\right|<\text { threshold }_{\text {amax }} \\
0 & \text { otherwise }\end{cases}
\end{aligned}
$$

2) The magnitude of the acceleration on z-axis $\left|a_{z k}\right|$ :

$$
C 2=\left\{\begin{array}{cc}
1 & \text { threshold }_{\text {azmin }}<\left|a_{z k}\right|<\text { threshold }_{\text {azmax }} \\
0 & \text { otherwise }
\end{array}\right.
$$

3) The magnitude of the angular velocity $\left|w_{k}\right|$ :

$$
\begin{aligned}
& \left|w_{k}\right|=\left[w_{x k}^{2}+w_{y k}^{2}+w_{z k}^{2}\right]^{1 / 2} \\
& C 3= \begin{cases}1 & \left|w_{y k}\right|<\text { threshold }_{\text {wmax }} \\
0 & \text { otherwise }\end{cases}
\end{aligned}
$$

4) The magnitude of the angular velocity on y-axis $\left|w_{y k}\right|$ :

$$
C 4=\left\{\begin{array}{cc}
1 & \left|w_{y k}\right|<\text { threshold }_{\text {ywmax }} \\
0 & \text { otherwise }
\end{array}\right.
$$

Since a foot firmly contacts the ground in a stance phase, the acceleration and rotation of the foot is zero over this duration. In an ideal condition the magnitude of total angular velocity $\left|w_{k}\right|$ in Equation (5c) and acceleration in a horizontal plane should be zero during a stance phase. However, the actual measurements are not zero due to vibration and the sensors' bias, but are still lower than the given thresholds.

By observing the outputs of a shoe-mounted IMU, it is found that the z-axis acceleration $a_{z k}$ and the y-axis angular velocity $w_{y k}$ are the most significant indicators 
in pedestrian travel. Due to the tilt of a shoe's surface, however, the measured $\left|w_{y k}\right|$ and $\left|a_{z k}\right|$ are not exactly 0 and g respectively. So the thresholds in Equations (5b) and (5d) are set based on the average value of z-axis accelerometer and y-axis gyro outputs during an initial stationary period, plus a certain level of fluctuation to ensure its robustness. The initial stationary period is at the beginning of sensor data collection when the shoe-mounted IMU is in a stationary condition.

2.4. Height Constraint. Similar to the concept proposed by Abdulrahim et al. (2012), we assume that the changes in height are only caused by climbing stairs since the floor is level in most indoor environments. If a change in height over one step is above a threshold, we regard it as reaching a new level or a stair; otherwise the height will be maintained at the previous level and uses this as a measurement of the Kalman filter. This constraint works well in structured environments with only level floor and stairs, but fails in the case of gently sloping terrains.

3. RUNNING. A stance phase detector for walking is incapable of detecting the stance phase correctly for running since the duration of the stance phase in running is shorter, and the stance phase is less stable as zero velocity. Barely enlarging the detection thresholds will introduce false stance phase detection.

In addition to developing a robust stance phase detector for walking and climbing as described in Section 2.3, we have also proposed a new detector for running. It includes a footstep detector that is used to determine the beginning of the gait cycle; a swing phase detector to indicate the swing phase of the step and zero velocity detectors that are used to detect the stance phase before the swing phase. The swing phase detector is used to avoid false detection during swing phase especially when the foot is nearly leaving the ground.

3.1. Footstep Detector. In pedestrian navigation with a shoe-mounted IMU, especially for running, it is important to know when a pedestrian takes a step. A footstep detector can not only mark the beginning of a gait cycle, but also helps to segment the pedestrian's gait cycle into four phases: stance, heel-off, swing, heel strike. A footstep detector mainly detects the heel strike after a foot completes swing phase in the air, reaches the highest point and touches down on the ground. Once the heel has hit the ground, the deceleration of the forefoot is very dramatic and characterised by large changes in the acceleration profile. Thus, we simplify the characteristic thus: in heel strike phase, the z-axis acceleration experiences a monotonic decreasing that is unique and obvious to detect so we denote it as a new step.

3.2. Swing Phase Detector. A swing phase detector is designed to identify swing phases and prevent false stance phase detection in swing phases when enlarging the thresholds of the stance phase detector for the running case. The swing phase detector introduced here is based on the energy of rotation threshold $T_{S} \cdot y_{k}^{g}$ is the angular velocity at the time instant $\mathrm{k}, \mathrm{W}$ is the window size. The swing phase detector is combined with the footstep detector to separate a swing phase from other phases. As swing phases are identified, false stance phase detection in swing phases can be avoided.

$$
T(k)=\frac{1}{W} \sum_{k}^{k+W-1}\left\|y_{k}^{g}\right\|^{2}<T_{s}
$$




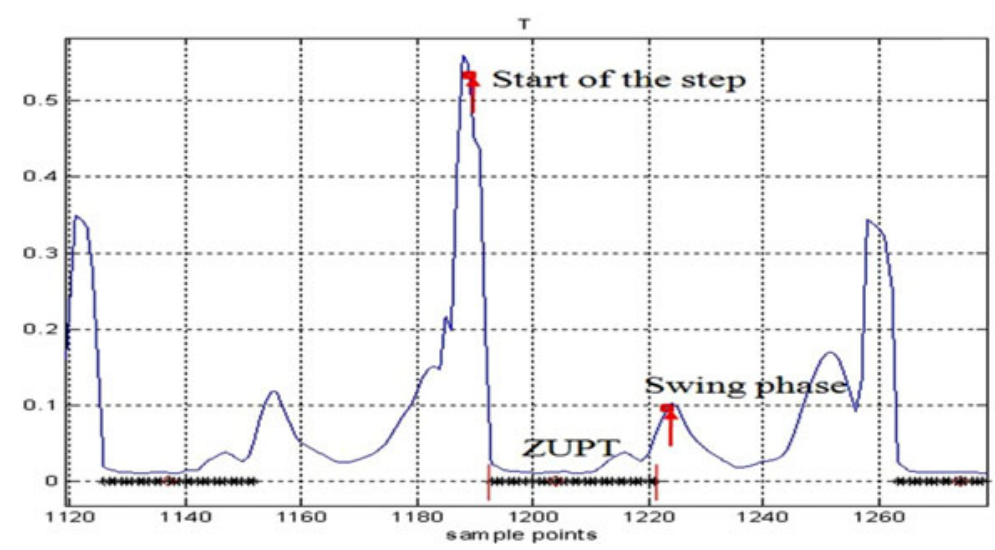

Figure 3. The energy of rotation $\mathrm{T}$.

Figure 3 shows the energy of rotation $\mathrm{T}$ in two gait cycles. It should be noted that the footstep detector can also use this $\mathrm{T}$ to detect heel strikes. The energy of rotation goes through a big jump when the foot falls down to the ground, as Figure 3 indicates.

4. CUPT. As mentioned by Abdulrahim et al. (2012), ZUPT will fail in two situations such as on an elevator or escalator. This is due to the fact that the stance phases detected on a moving platform are not zero velocity. If a ZUPT algorithm is applied to a moving platform, it will regard the moving platform as having zero velocity and perform ZUPT. As a result, the navigation solution is obviously wrong. Use of other sensors or additional measurements may help to address these issues, but it will be complex and costly. Without additional sensors, we have proposed a CUPT algorithm that uses constant velocity as the additional measurement.

ZUPT is just a special case in CUPT where the true velocity in the stance phases is zero. CUPT can detect the stance phases as ZUPT when stepping on the ground, and also a constant velocity when the pedestrian is standing in a moving elevator or on an escalator.

The core concept of the CUPT algorithm is to detect the velocity of a platform instead of assuming it as zero as in ZUPT. Obviously, the stance phase detection on a moving platform is exactly the same as the stance phase detection on the ground without considering a platform velocity. However, CUPT needs not only to identify a stance phase, but also to measure the velocity of the moving platform and decide whether the velocity is constant or not.

4.1. Elevator. Taking an elevator as example, Figure 4 shows the measurement of the vertical accelerometer $\mathrm{Acc}_{\mathrm{Z}}$ and the estimated velocity. A pedestrian walked into the elevator and stood still when the elevator was moving. As indicated by the velocity in Figure 4, the elevator went through a period of acceleration, kept at constant velocity and decelerated before it was stationary again. The constant velocity appears once the elevator finishes accelerating and before the elevator starts slowing down. In this circumstance, the most significant indicator is the acceleration in the vertical direction. 


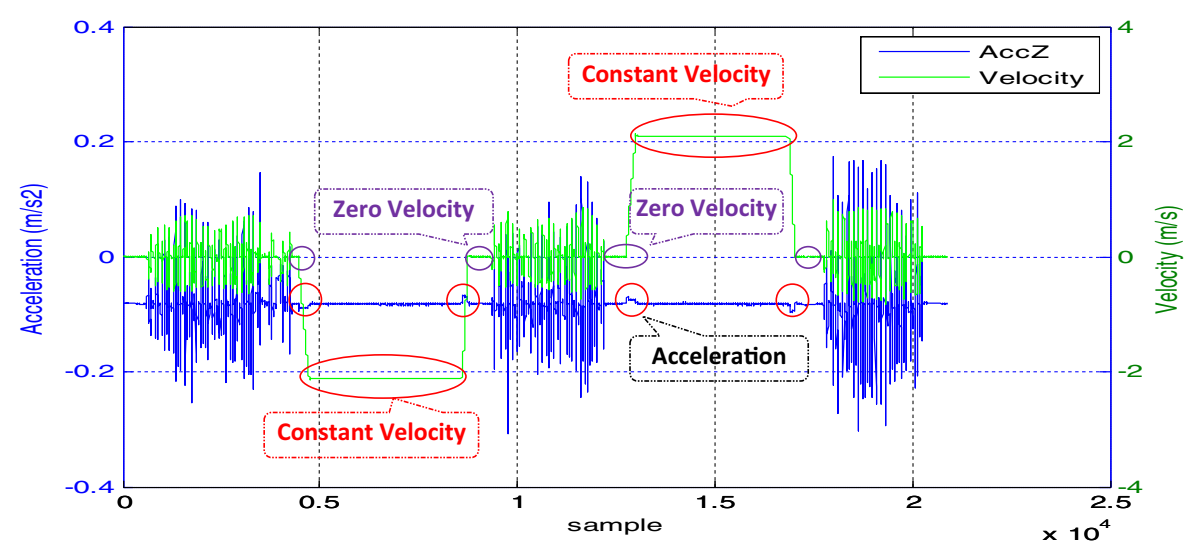

Figure 4. Indication of motion in an elevator.

When the elevator starts to move, the acceleration in the z-axis experiences a jump which can be easily distinguished from the normal gaits. When this special case of acceleration has been detected, the velocity is calculated by the inertial navigation algorithm without a velocity measurement update. Since the pedestrian stands in the elevator, the zero horizontal velocity can be added as a measurement to the EKF, referencing to Equation (4):

$$
z=\hat{x}-v_{X Y}
$$

After the elevator finishes accelerating, the velocity keeps constant before the elevator begins to slow down. During this constant velocity section, CUPT is applied to correct navigation errors. So the measurement $\mathrm{z}$ is $\hat{x}-v_{\text {constant }}$. The decelerating section is similar to the accelerating section, and the zero horizontal velocity update is applied.

During the accelerating and decelerating sections, elevator data processing follows the INS mechanization to obtain position, velocity and attitude information without any velocity update until a constant velocity is detected. However, in some cases it is hard to detect constant velocity. The motion in accelerating and decelerating sections actually has a variable acceleration rate. Especially during braking, an elevator first slows down to a low speed, then keeps at this low constant velocity for a while, before decelerating to zero velocity. As a result, frequent elevator stops have an influence on the accuracy of height measurement.

4.2. Escalator. For the case of being on an escalator, the horizontal velocity is the most significant indicator. When a foot steps onto the escalator and keeps pace with the escalator going up or down, a constant velocity is determined by the instance of the foot being set on the escalator. So in this case, the measurement $\mathrm{z}$ is $\hat{x}-v_{\text {constant }}$. Once the horizontal velocity is over a threshold in stance phase, it is assumed that the foot is on an escalator and we apply CUPT as measurement of the EKF.

Figure 5 shows the horizontal and vertical velocity of a foot on a moving escalator. We can see that ZUPT is applied when the pedestrian walks on the ground; the observation is zero velocity during the stance phase, while the velocity keeps constant when the pedestrian stands on the escalator and CUPT corrects the velocity error using the constant velocity as the observation. 


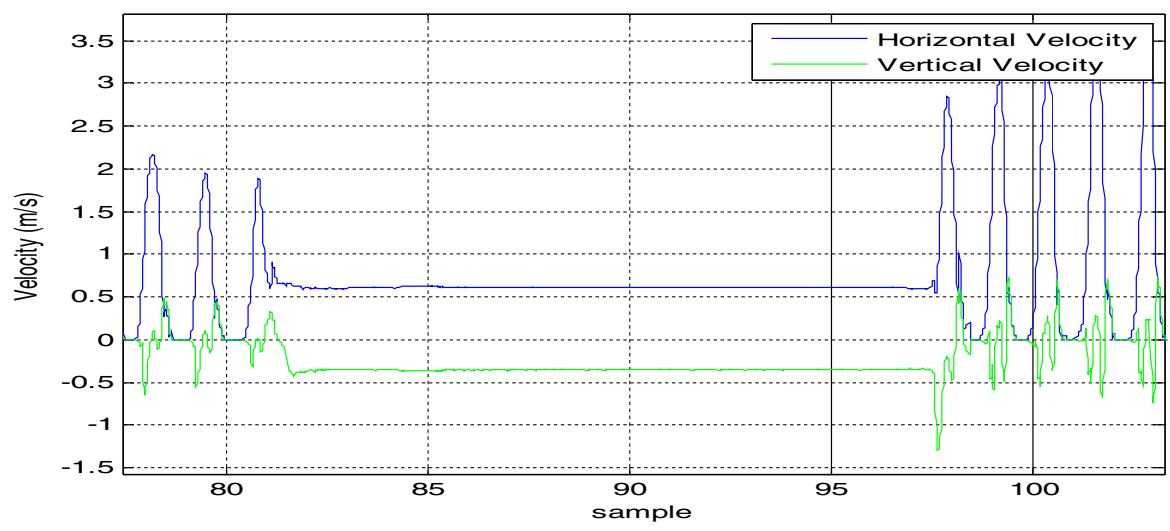

Figure 5. Motion in an escalator with CUPT.

In summary, the stance phase detection for CUPT is the same with ZUPT since the foot is statically in contact with a platform in both cases. But for CUPT the velocity of the moving platform needs to be measured. ZUPT is applied when a person is walking on the ground while CUPT is to handle the case on a moving platform. In order to apply CUPT, firstly we need to use the stance phase detector to declare whether the foot is stationary, then determine whether the foot is on a moving platform, measure the constant velocity of the platform and apply CUPT to correct the errors.

5. SMOOTHING. Smoothing uses the fact that the sensor data can also be used in a reverse time fashion. The goal of smoothing is to determine an optimal estimate utilizing information before and after the current time. This paper introduces a closed-loop step-wise smoothing algorithm based on a Rauch-Tung-Striebel (RTS) smoother.

5.1. RTS Smoother. The RTS smoother is a two-pass smoothing algorithm. The forward pass is the same as the regular Kalman filter algorithm. During the prediction and update steps, the states and covariance are saved for use in the backwards pass. After the forward pass, the RTS smoothing begins from the end of the mission and moves back to the starting point of the data set. In the backwards pass, the smoothed state estimates and covariance propagate using the states saved in the forward pass (Rauch et al., 1965). The smoothed estimate of the states is computed using the equations below.

$$
\begin{gathered}
\hat{x}_{k \mid N}=\hat{x}_{k \mid k}+C_{k}\left[\left(\hat{x}_{k+1 \mid N}-\hat{x}_{k+1 \mid k}\right]\right. \\
P_{k \mid N}=P_{k \mid k}+C_{k}\left(P_{k+1 \mid N}-P_{k+1 \mid k}\right) C_{k}^{\prime}
\end{gathered}
$$

where $C_{k}=P_{k \mid k} \emptyset^{\prime(k+1, k) P_{k+1 \mid k}^{-1}}, \hat{x}_{k \mid k}, \hat{x}_{k+1 \mid k}, P_{k \mid k}, P_{k+1 \mid k}$ are the error states and their variances stored in the forward filter.

5.2. Data Segmentation. The implementation of the RTS smoother has some limitations. Firstly, it is commonly applied for post processing. Secondly, a lot of memory is required to store the predicted and updated information (Chiang et al., 2012). In order to achieve near-real-time smoothing and reduce the storage burden, 
one intuitive way is to take profit of the detected zero velocity intervals and divide the step with them.

In order to get near-real-time smoothing, proper segmentation rules should be applied. The principle for our data segmentation is similar to the one proposed by Colomar et al. (2012). They use velocity error covariance as a threshold. The choice of the threshold value is based on typical values from the training data of random walking, so it is not suitable for scenarios that involve running. Moreover, it is not applicable for navigation on a moving platform, such as elevator or escalator, where the velocity of a foot in stance phase may not converge to zero but a constant velocity. In addition, the fixed time constant of 30 samples that they applied may work properly in normal walking but fail occasionally in running, since the duration of stance phase in running may be shorter than 30 samples and it will cause misdetection. Furthermore, if the person alternates between walking and running, the segmentation rule based on velocity error covariance will easily get confused and become invalid.

We proposed a new segmentation rule by combining both the footstep detector and swing phase detector. The energy of rotation threshold $\mathrm{T}$ is used to avoid false segmentation in swing phases, which makes the rule more flexible to meet the complex environment.

The segmentation point should be in the stance phase. Here we have tried three methods to get the segmentation point; the first one is to use the middle point of the stance phase, the second one is the sample point with smallest $\mathrm{T}$ during the stance phase and the third one is the sample point with $\mathrm{T}$ under a certain small threshold. According to our tests, these three methods have little difference in the final smoothing results, which means that we do not need to find where the exact point converges to zero, as long as the segmentation point is within the stance phase. The third method is used as the segmentation rule in the following closed-loop smoothing.

5.3. Closed-Loop Smoothing. In our ZUPT-aided INS implementation, the predicted EKF error states are always reset to zero. During measurement updates, the

\begin{tabular}{|c|c|c|}
\hline Time Update & $\begin{array}{l}\text { INS mechanization } \\
\text { Error states and error covariance propagate }\end{array}$ & $\begin{array}{l}\hat{\mathrm{x}}_{\mathrm{k}}^{-} \\
\delta \hat{\mathrm{x}}_{\mathrm{k}}^{-}=\mathrm{F}_{\mathrm{k} \cdot \mathrm{k}-1} \delta \hat{\mathrm{x}}_{\mathrm{k}-1}^{+} \\
\mathrm{P}_{\mathrm{k}}^{-}=\mathrm{F}_{\mathrm{k} \cdot \mathrm{k}-1} \mathrm{R}_{\mathrm{k}-1}^{+} \mathrm{F}_{\mathrm{k} k-1}^{\mathrm{T}}+\mathrm{Q}_{\mathrm{k}-1}\end{array}$ \\
\hline Footstep detector & Detect start of the step & $\mathrm{T}\left(\mathrm{k}_{1}\right)<\mathrm{T} 1$ \\
\hline $\begin{array}{l}\text { Measurement Update } \\
\text { If ZUPT }\end{array}$ & $\begin{array}{l}\text { Error states and error covariance update } \\
\text { Compensate navigation states }\end{array}$ & $\begin{array}{l}\mathrm{K}_{\mathrm{k}}=\mathrm{P}_{\mathrm{k}}^{-} \mathrm{H}_{\mathrm{k}}^{\mathrm{T}}\left(\mathrm{H}_{\mathrm{k}} \mathrm{P}_{\mathrm{k}}^{-} \mathrm{H}_{\mathrm{k}}^{\mathrm{T}}+\mathrm{R}_{\mathrm{k}}\right)^{-1} \\
\mathrm{v}_{\mathrm{k}}=\mathrm{z}_{\mathrm{k}}-\mathrm{H}_{\mathrm{k}} \delta \hat{\mathrm{x}}_{\mathrm{k}}^{-} \\
\delta \hat{\mathrm{x}}_{\mathrm{k}}^{+}=\delta \hat{\mathrm{x}}_{\mathrm{k}}^{-}+\mathrm{K}_{\mathrm{k}} \mathrm{v}_{\mathrm{k}} \\
\mathrm{P}_{\mathrm{k}}^{+}=\left(\mathrm{I}-\mathrm{K}_{\mathrm{k}} \mathrm{H}_{\mathrm{k}}\right) \mathrm{P}_{\mathrm{k}}^{-} \\
\hat{\mathrm{x}}_{\mathrm{k}}^{+}=\hat{\mathrm{x}}_{\mathrm{k}}^{-}+\delta \hat{\mathrm{x}}_{\mathrm{k}}^{+}\end{array}$ \\
\hline Footstep detector & Detect swing phase & $\mathrm{T}\left(\mathrm{k}_{2}\right)<\mathrm{T} 2$ \\
\hline Segmentation & $\begin{array}{l}\text { Segmentation rule } \\
\text { Decide: go back to time update/ leave loop }\end{array}$ & $\mathrm{k}_{1}<\mathrm{k}<\mathrm{kT}(\mathrm{k})<\mathrm{T} 3$ \\
\hline After loop & $\begin{array}{l}\text { Smoothing of the error states } \\
\text { Compensate navigation states } \\
\text { Go back to loop and go on processing }\end{array}$ & $\begin{array}{l}\delta \hat{\mathrm{x}}_{\mathrm{k} \mid \mathrm{N}} \quad(\text { Equation 9) } \\
\hat{\mathrm{x}}_{\mathrm{k} \mid \mathrm{N}}=\hat{\mathrm{x}}_{\mathrm{k}}{ }^{+}+\delta \hat{\mathrm{x}}_{\mathrm{k} \mid \mathrm{N}}\end{array}$ \\
\hline
\end{tabular}

Figure 6. Step wise close loop smoothed ZUPT aided INS. 
Table 1. Specification of Navchip IMU.

\begin{tabular}{llll}
\hline Gyroscope Performance & & Accelerometer Performance & \\
\hline Range $(\mathrm{deg} / \mathrm{sec})$ & \pm 480 & Range $\left(\mathrm{m} / \mathrm{s}^{2}\right)$ & \pm 8 \\
Noise Density $(\% / \mathrm{s} / \sqrt{ } \mathrm{Hz})$ & $0 \cdot 004$ & Noise Density $(\mathrm{ug} / \sqrt{ } \mathrm{Hz})$ & 70 \\
Bias Stability $(\% / \mathrm{hr}, 1 \sigma)$ & 12 & Bias Stability $(\mathrm{mg}, 1 \sigma)$ & $0 \cdot 1$ \\
\hline
\end{tabular}

estimated error states are corrected and fed back to compensate the estimated navigation states. However, in a closed-loop smoothing-aided ZUPT, the EKF runs as normal but the predicted error states need to be propagated and no longer reset to zero. The estimated states and covariance are updated only when ZUPT are applied and the smoothing is applied over the error states. After the step segmentation is performed, the estimated states are combined with the smoothed error states to get the final navigation results. Figure 6 summarises the implementation procedures for the closed-loop smoothing.

6. EXPERIMENTS. The proposed pedestrian navigation system has been tested for walking, running, stair climbing and taking an elevator or escalator.

6.1. Hardware Description. We implemented our pedestrian navigation system with the Navchip IMU from Intersense. The small size of Navchip makes it easy to attach to a boot and has no effect on walking. Table 1 lists the specification of Navchip (encapsulated within casing).

6.2. Walking and Running Test.

6.2.1. Experimental data processed results. All the trajectories are closed-loop with the finish point the same as the start point. Since the start and stop points are the exact same location in reality, the distance between the black and blue dots in the below figures represents the error on the horizontal plane. Figure 7 represents three trajectories including stair climbing.

Table 2 shows the final return position errors for three-dimensional (3D) experiments.

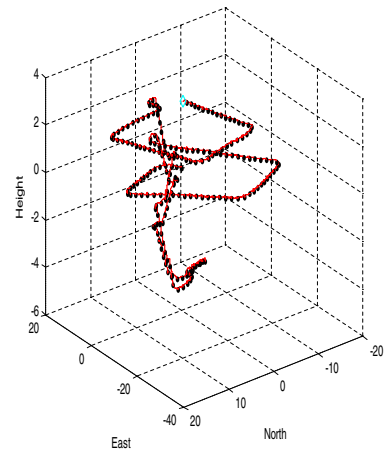

(a)

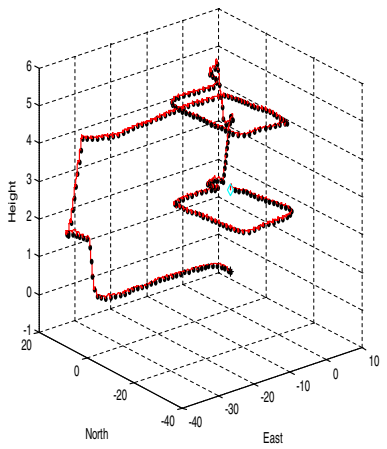

(b)

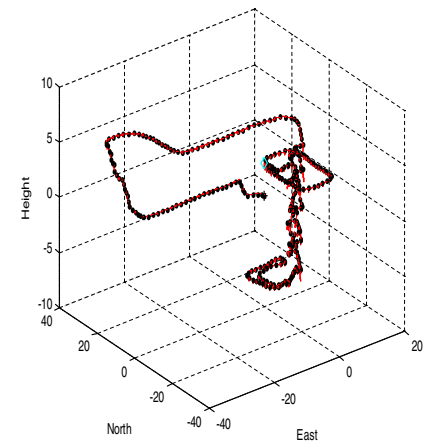

(c)

Figure 7 (a) 3D trajectory 1, (b) 3D for trajectory 2 and (c) 3D trajectory 3. 
Table 2. Return Position Errors.

\begin{tabular}{lccc}
\hline & & \multicolumn{2}{c}{ Relative Position Error (\%) } \\
\cline { 2 - 4 } Test & Estimated Distance (m) & X-Y plane & Z direction \\
\hline 3D Walk 1 & $354 \cdot 8$ & $0 \cdot 30 \%$ & $0 \cdot 61 \%$ \\
3D Walk 2 & $240 \cdot 8$ & $0 \cdot 14 \%$ & $0 \cdot 70 \%$ \\
3D Walk 3 & $277 \cdot 4$ & $0 \cdot 01 \%$ & $0 \cdot 71 \%$ \\
3D Walk 4 & $339 \cdot 2$ & $0 \cdot 08 \%$ & $0 \cdot 90 \%$ \\
3D Walk 5 & $408 \cdot 6$ & $0 \cdot 75 \%$ & $0 \cdot 25 \%$ \\
Average & $\mathbf{3 2 4 \cdot 2}$ & $\mathbf{0 \cdot 2 6} \%$ & $\mathbf{0 \cdot 6 3} \%$ \\
\hline
\end{tabular}

Table 3. Return Position Errors.

\begin{tabular}{llccc}
\hline \multirow{2}{*}{ Test } & & \multicolumn{2}{c}{ Relative Position Error (\%) } \\
\cline { 3 - 4 } Trajectory1 & & Estimated Distance $(\mathrm{m})$ & X-Y plane & Z direction \\
\cline { 4 - 5 } & Walk & $292 \cdot 1$ & $0 \cdot 24 \%$ & $1 \cdot 24 \mathrm{e}-4 \%$ \\
\multirow{2}{*}{ Trajectory2 } & Walk \& run & $173 \cdot 6$ & $0 \cdot 96 \%$ & $8 \cdot 5 \mathrm{e}-5 \%$ \\
& Run & $67 \cdot 9$ & $0 \cdot 42 \%$ & $0 \cdot 002 \%$ \\
\multirow{2}{*}{ Average } & Walk & $195 \cdot 7$ & $0 \cdot 20 \%$ & $4 \cdot 54 \mathrm{e}-4 \%$ \\
& Run & $173 \cdot 2$ & $0 \cdot 95 \%$ & $8 \cdot 66 \mathrm{e}-5 \%$ \\
\hline
\end{tabular}

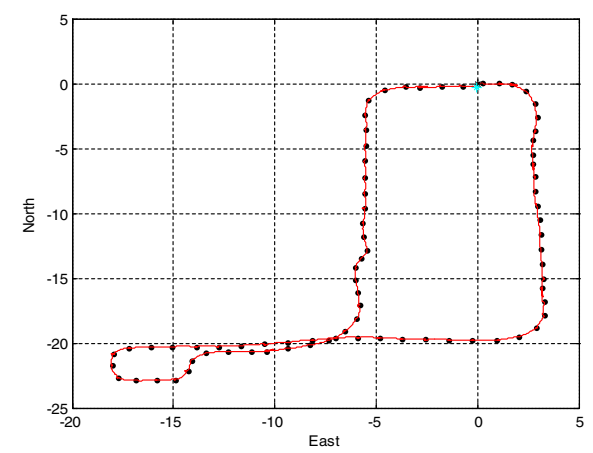

(a)

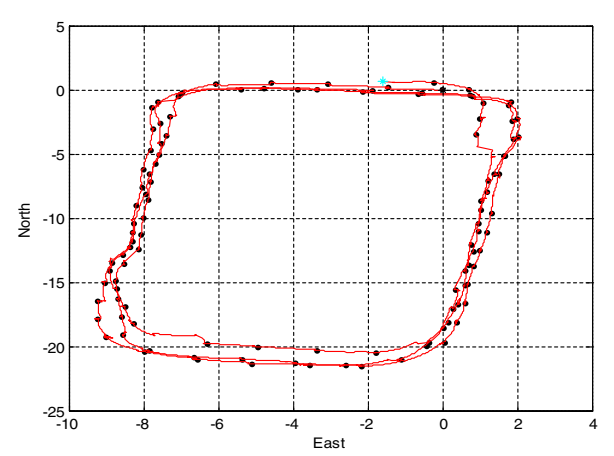

(b)

Figure 8 (a) Trajectory 1 and (b) Trajectory 2.

In this section we present two trajectories (Figure 8) and each trajectory is traversed several times with different human gaits that include walking, running and alternating between walking and running. All the trajectories are closed-loop.

Table 3 summarises the final return position errors for each trajectory and Table 4 also gives the comparison results of different gait styles.

Ideally the error of running should be larger than that of alternating between walking and running, since the higher dynamics experienced during running than in walking result in fewer ZUPT applications. However, from 
Table 4. Errors of Different Gait styles.

\begin{tabular}{lcc}
\hline & \multicolumn{2}{c}{ Relative Position Error (\%) } \\
\cline { 2 - 3 } Gait style & X-Y plane & X-Y plane \\
\hline Walk \& run & $0 \cdot 96 \%$ & $8 \cdot 58 \mathrm{e}-5 \%$ \\
Run & $0 \cdot 72 \%$ & $0.0018 \%$ \\
\hline
\end{tabular}

Table 5. Distance-Travelled Errors Normalized to the Total Distance Travelled.

\begin{tabular}{cccc}
\hline & Data Set & Distance Travelled (m) & Positioning Error (\%) \\
\hline Walk "Walk 2D - Rectangle other direction" & $28 \cdot 68$ & $0 \cdot 050 \%$ \\
& "Walk 2D - 8" & $47 \cdot 46$ & $0 \cdot 049 \%$ \\
& "Walk 2D - On table" & $24 \cdot 42$ & $0 \cdot 43 \%$ \\
& "Walk 2D - Patrick long" & $2 \cdot 79 \mathrm{e} 2$ & $0 \cdot 10 \%$ \\
Run & "Run 2D - Straight' & $28 \cdot 34$ & $0 \cdot 13 \%$ \\
& "Run 2D - Circle" & $73 \cdot 94$ & $0 \cdot 26 \%$ \\
& "Run 2D - Circle other direction" & $40 \cdot 41$ & $0 \cdot 20 \%$ \\
\hline
\end{tabular}

our results as shown in Table 4, we get a different conclusion. The main reason is that when the pedestrian changes his gait randomly, sometimes it is easy to confuse the footstep detector. On the other hand, the swing phase detector is applied to ensure that no false stance phase detection in swing phases, thus we can still get reasonable results.

In addition, out of the excessive shock of feet strikes, the bi-directional overflows exceed the ranges of accelerometer and/or gyroscope and will affect navigation accuracy to a large extent. Although we have already taken measures to reduce the impact, it is still inevitable that the overflow would affect the INS mechanism computing vertical velocity and position.

6.2.2. Reference data processed results. In this section, we also give the results by processing the reference dataset provided in Angermann et al. (2010). In their paper, the authors also give the processed results using two customary methods that fail when processing the running data. Table 5 shows the distance travelled error normalized to the total distance travelled.

6.3. Elevator and Escalator Test. This section describes elevator and escalator tests conducted in the UTS tower building.

6.3.1. Elevator test. The pedestrian walked for a while before going into an elevator. The pedestrian randomly chose which elevator to take and which level to stop at. When the door was closed, the pedestrian stopped walking and stayed still until the door opened again.

Three trajectories are shown in Figure 9. The left figure is the $3 \mathrm{D}$ view of the trajectory while the right figure represents the height change over time shown by the red line. The black dots in the right figures indicate the sample points have been applied CUPT in the slope sections with a constant velocity, or ZUPT in the flat sections. It is noticed that only a few small sections of the red line in the right figures 

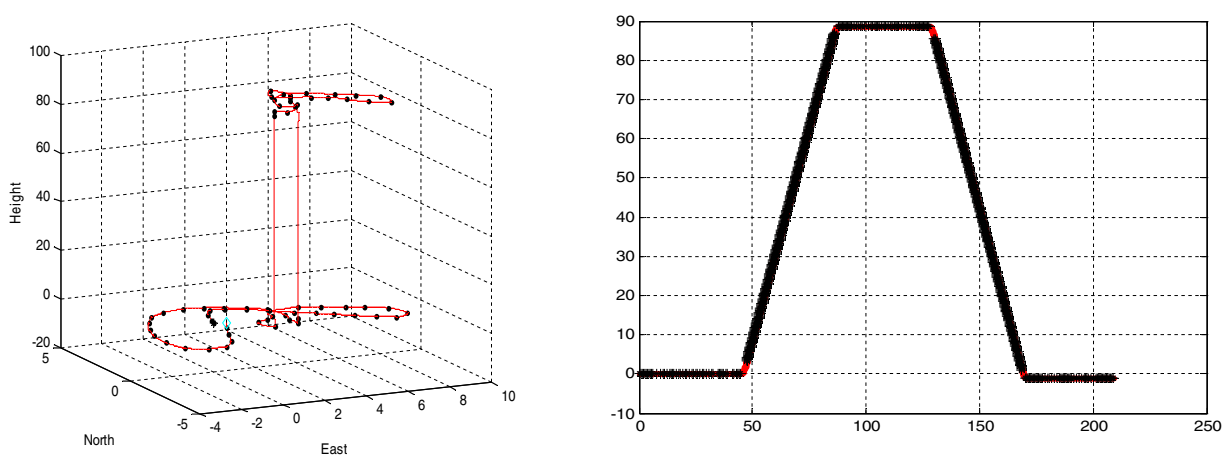

(a)
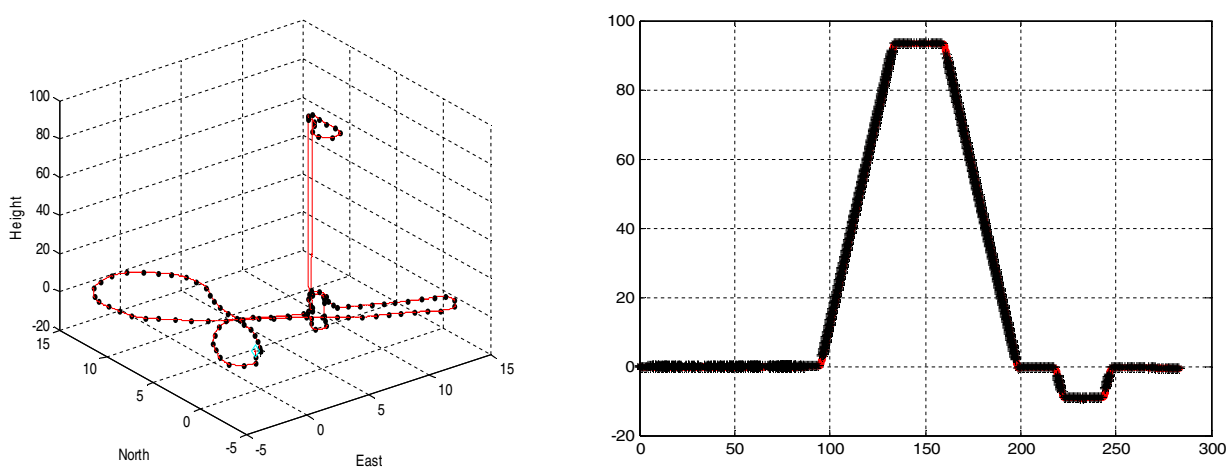

(b)
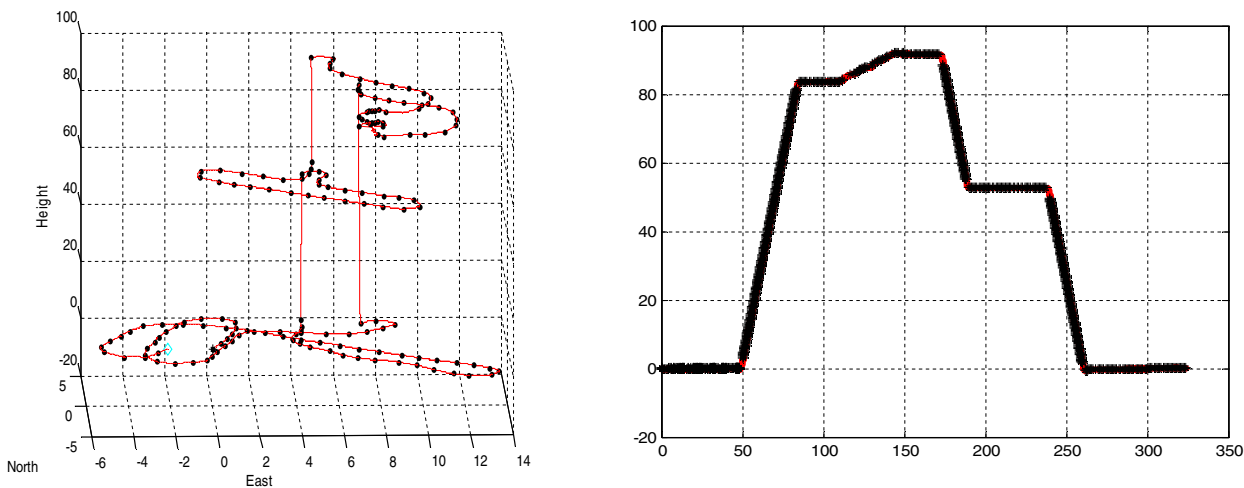

(c)

Figure 9 (a) Trajectory 1 of elevator test, (b) Trajectory 2 of elevator test and (c) Trajectory 3 of elevator test.

are not covered by the black dots, which are the periods that the elevator is accelerating or decelerating where neither ZUPT nor CUPT can be applied for vertical position and speed correction. 

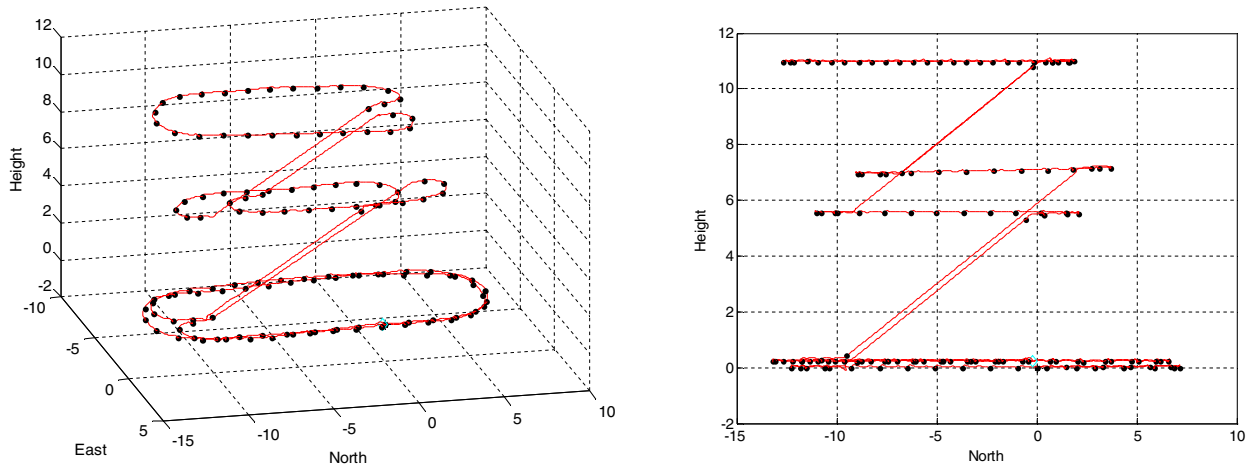

Figure 10. Trajectory of escalator test.

Table 6. Return Position Errors for Elevator.

\begin{tabular}{lccc}
\hline & & \multicolumn{2}{c}{ Relative Position Error (\%) } \\
\cline { 3 - 4 } Elevator & Estimated Travel Distance $(\mathrm{m})$ & $\mathrm{X}-\mathrm{Y}$ plane & $\mathrm{Z}$ direction \\
\hline Trajectory 1 & $248 \cdot 3$ & $0 \cdot 17 \%$ & $0 \cdot 47 \%$ \\
Trajectory 2 & $324 \cdot 7$ & $0 \cdot 39 \%$ & $0 \cdot 063 \%$ \\
Trajectory 3 & $373 \cdot 0$ & $0 \cdot 56 \%$ & $0 \cdot 02 \%$ \\
Average & $\mathbf{3 1 5 \cdot 3}$ & $\mathbf{0 \cdot 3 7 \%}$ & $\mathbf{0 \cdot 1 8} \%$ \\
\hline
\end{tabular}

Table 7. Return Position Errors for Escalator.

\begin{tabular}{lccc}
\hline & & \multicolumn{2}{c}{ Relative Position Error (\%) } \\
\cline { 3 - 4 } Escalator & Estimated Travel Distance $(\mathrm{m})$ & $\mathrm{X}-\mathrm{Y}$ plane & Z direction \\
\hline Trajectory 1 & $223 \cdot 3$ & $0 \cdot 34 \%$ & $0 \cdot 11 \%$ \\
Trajectory 2 & $259 \cdot 1$ & $0 \cdot 56 \%$ & $0 \cdot 34 \%$ \\
Trajectory 3 & $232 \cdot 4$ & $0 \cdot 64 \%$ & $0 \cdot 37 \%$ \\
Trajectory 4 & $210 \cdot 8$ & $0 \cdot 22 \%$ & $0 \cdot 10 \%$ \\
Average & $\mathbf{2 3 1 \cdot 4}$ & $\mathbf{0 \cdot 4 4} \%$ & $\mathbf{0 \cdot 2 3} \%$ \\
\hline
\end{tabular}

6.3.2. Escalator test. Tests were also conducted for an escalator. As shown in Figure 10, starting at a lower level, the pedestrian walked for a while and then stood on a rising escalator with the IMU mounted on one foot. After getting off the escalator at the middle level the pedestrian walked to another rising escalator to the top level; and then walked to the two falling escalators in sequence to descend back to the start point.

Figure 10 is the 3D view of the trajectory, which clearly represents the four escalators and the three levels walked. Tables 6 and 7 summarise the return position errors for elevator and escalator experiments respectively. 
Although these test results are reasonably good, there is still plenty of room to further improve the proposed algorithm to achieve better performance and handle more challenging situations. For the escalator, the vertical angle of the escalators was predetermined to compute the vertical velocity. This angle could be measured by the sensor with more sophisticated investigation. Although the height relative error in Table 7 is small, the horizontal relative error is not so good. This may be due to the change in the vertical angle at the two end sections of escalators where the staircase keeps moving forward but does not change in the vertical direction. The predetermined slope angle introduces error for the measurement update in the short end sections.

6.4. Smoothing Results. All experimental tests have been conducted in UTS, including walking, running and taking escalator and elevator. The figures below show the achieved smoothing effect compared with the estimated trajectory.

Figures 11 and 12 show the effect of smoothing over a walking and running trajectory respectively. From the height and velocity analysis, we can see that when the first ZUPT is applied, the correction applied over the estimated trajectory results in a big jump appearing in every step. After smoothing, these big jumps can be eliminated thus the trajectory is smoother than the original one.

Compared with walking, the magnitude of running velocity may vary and not converge to exactly zero as walking does during the stance phase.

For the cases in an elevator and on an escalator, CUPT is applied to let velocity not converge to zero as ZUPT, but to a constant velocity when the pedestrian stands on the moving platform. It is also obvious to see that the smoothing
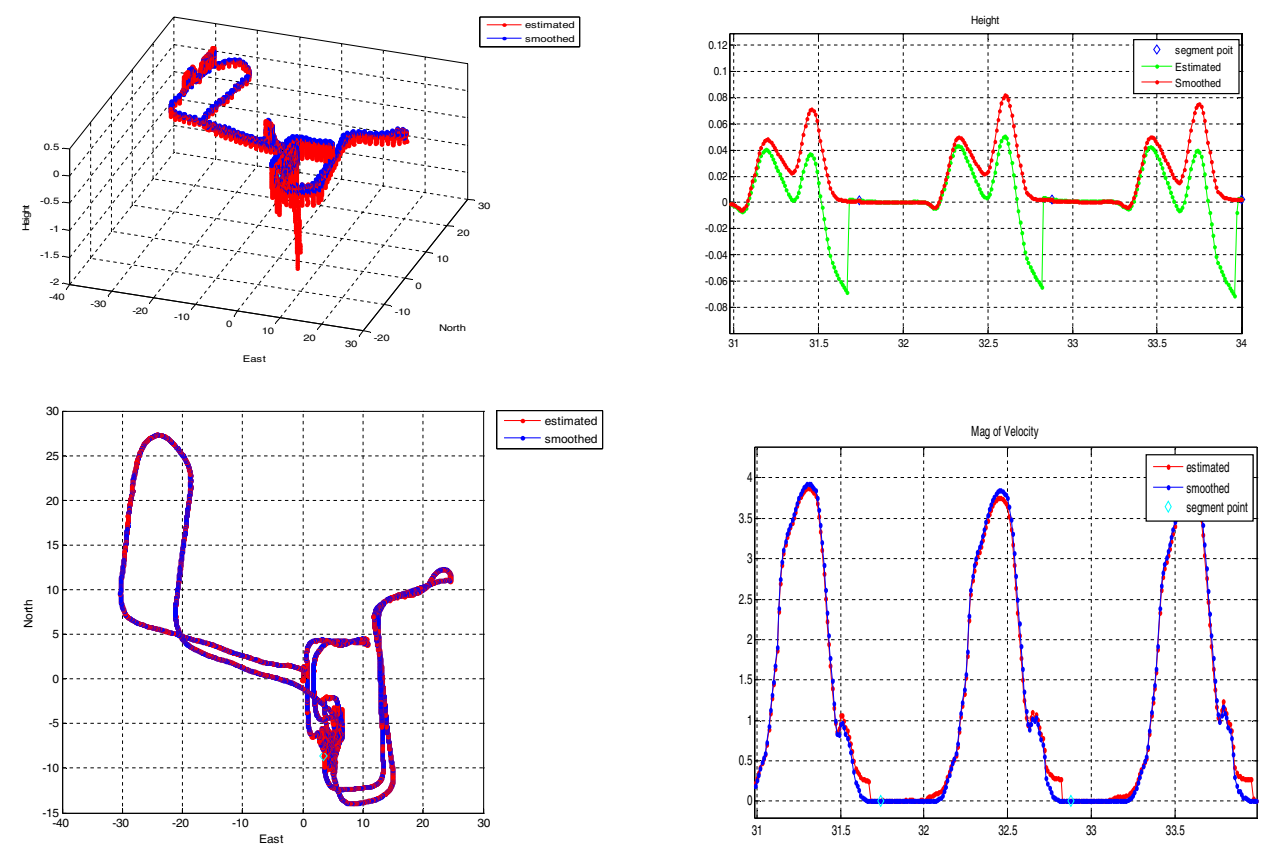

Figure 11. Effect of smoothing on a walking trajectory. 

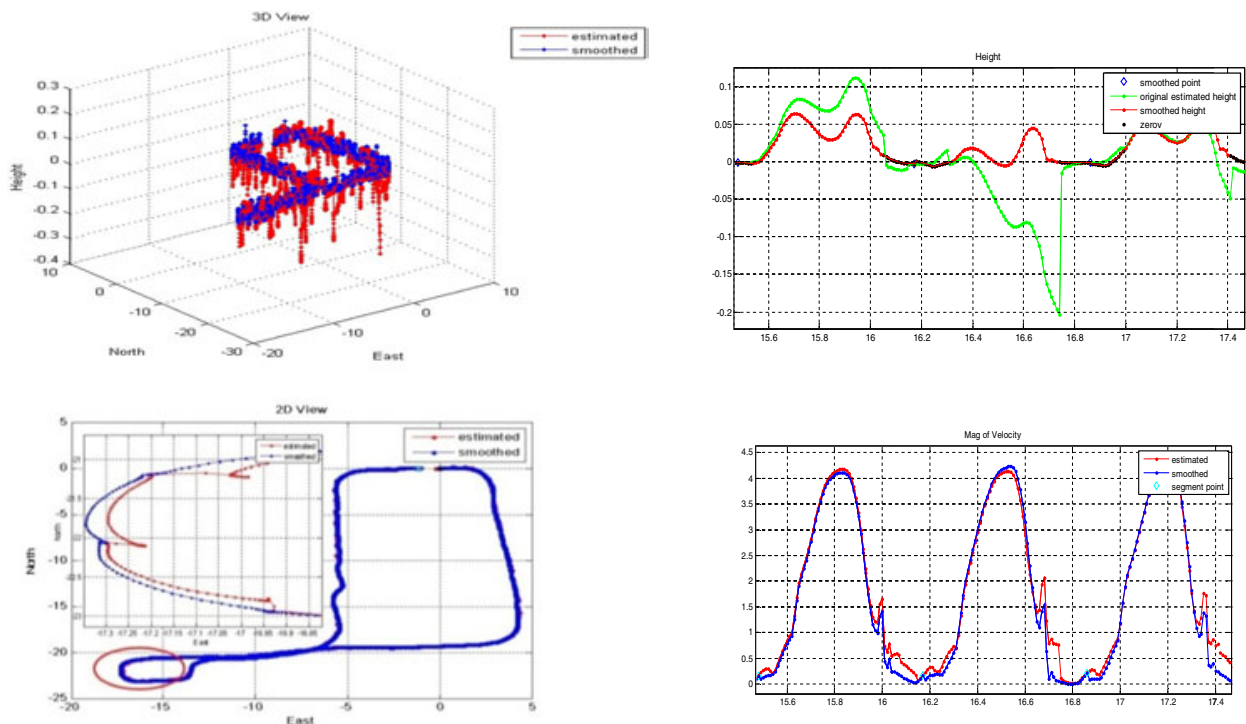

Figure 12. Effect of smoothing on a running trajectory.

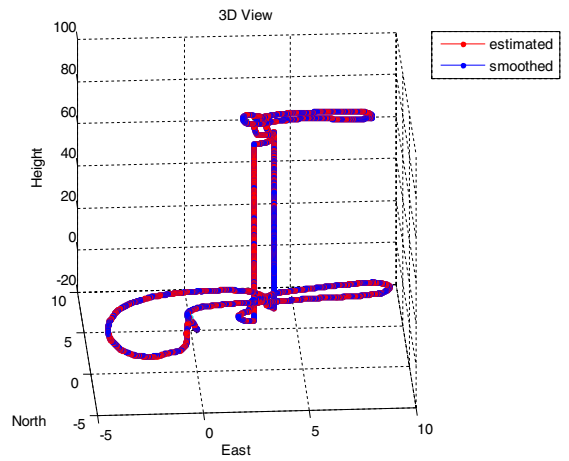

20 View
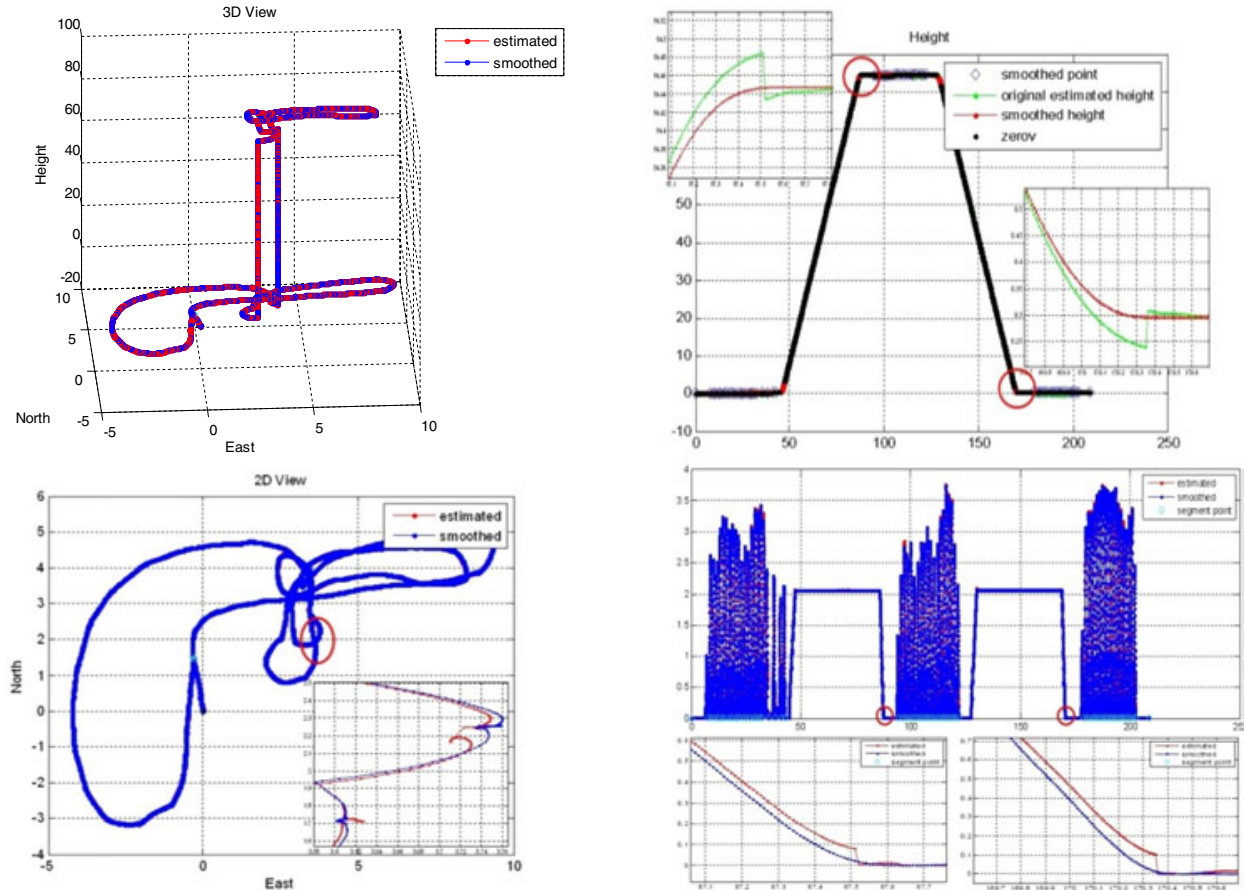

Figure 13. Effect of smoothing on an elevator trajectory.

algorithm can significantly improve the smoothness of the trajectory especially when the elevator finishes accelerating or decelerating and the pedestrian gets on or off the escalator (Figures 13 and 14). 

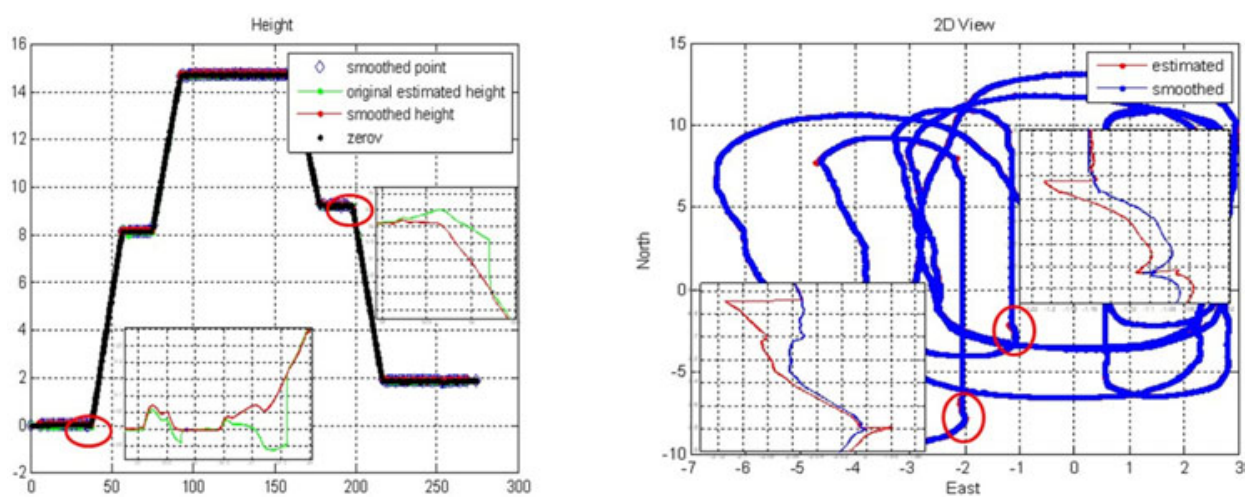

Figure 14. Effect of smoothing on an escalator trajectory.

7. CONCLUSION. In this paper, we have documented a robust pedestrian navigation system that can not only detect the stance phase for running, but also give the navigation solution when the pedestrian is in an elevator and on an escalator. Based on the RTS smoother, we also proposed a closed-loop step-wise smoothing to eliminate the sharp correction and discontinuity of the trajectory.

This paper presented a self-contained pedestrian navigation system that could work in both $2 \mathrm{D}$ and $3 \mathrm{D}$ environments with good performance. For 3D stairs climbing experiments, the average final return position error on the horizontal plane is around $0 \cdot 26 \%$ of distance travelled, the height drift is about $0.63 \%$, while the final return position error for $2 \mathrm{D}$ walking is even smaller, that is $0 \cdot 16 \%$ on $\mathrm{X}-\mathrm{Y}$ plane by average and we can always get back to the original position for each loop with an average error of $0 \cdot 33 \%$.

The proposed pedestrian navigation system can also handle running cases. From the test results, we can draw a conclusion that the algorithm is efficient to limit the growth of IMU error. Our robust stance phase detection shows satisfactory performance.

In addition, this paper presents a CUPT algorithm that works on a moving platform with good performance. For elevator experiments, the average return position error on the horizontal plane is around $0.37 \%$ of the total distance travelled; the height drift is about $0 \cdot 18 \%$. The return position error for an escalator is a little larger, that is $0 \cdot 44 \%$ in the horizontal plane by average and $0.23 \%$ in the vertical.

Future work will attempt to address several aspects of the navigation system. Firstly, more specific mathematical models will be analysed for the elevator and escalator tests. Secondly, augmentation with other sensors will be explored, such as using a magnetometer to improve the navigation accuracy. Finally, the feasibility of integration with GPS and a camera will be tested. This multi-sensor integration will make the pedestrian navigation system meet more complex environments and thus broadening the practical use.

\section{REFERENCES}

Aoki, H., Schiele, B. and Pentland, A. (1999). Real-time personal positioning system for wearable computer. The 3rd IEEE International Symposium on Wearable Computers (ISWC 1999), Washington, DC, USA. 
Abdulrahim, K., Hide, C., Moore, T. and Hill, C. (2012). Using constraints for shoe mounted indoor pedestrian navigation. Journal of Navigation. 65(1), 15-28.

Angermann, M., Robertson, P., Kemptner, T. and Khider, M. (2010). A high precision reference data set for pedestrian navigation using foot-mounted inertial sensors. 2010 IEEE International Conference on Indoor Positioning and Indoor Navigation (IPIN), Zurich, Switzerland, 1-6.

Bebek, O., Suster, M.A., Rajgopal, S., Fu, M.J., Huang, X., Çavuşoğlu, M.C. and Mastrangelo, C.H. (2010). Personal navigation via high-resolution gait-corrected inertial measurement units. The IEEE Transactions on Instrumentation and Measurement, 59(11), 3018-3027.

Castaneda, N. and Lamy-Perbal, S. (2010). An improved shoe-mounted inertial navigation system. 2010 International Conference on Indoor Positioning and Indoor Navigation (IPIN), Campus Science City, ETH Zurich.

Chiang, K.W., Duong, T.T., Liao, J.K., Lai, Y.C., Chang, C.C., Cai, J.M. and Huang, S.C. (2012). On-line smoothing for an integrated navigation system with low-cost MEMS inertial sensors. Sensors 12(12), 17372-17389.

Cho, S.Y., Lee, K.W., Park, C.G. and Lee, J.G. (2003). A personal navigation system using low-cost MEMS/GPS/Fluxgate. The Proceedings of the 59th Annual Meeting of the Institute of Navigation and CIGTF 22nd Guidance Test Symposium, Albuquerque, NM.

Cho, S.Y. and Park, C.G. (2006). MEMS based pedestrian navigation system. Journal of Navigation, 59(1), 135-153.

Colomar, S.D., Nilsson, J.O., Handel, P. (2012). Smoothing for ZUPT-aided INSs. 2012 IEEE International Conference on Indoor Positioning and Indoor Navigation (IPIN), Sydney, Australia.

Feliz, R., Zalama, E. and García-Bermejo, J.G. (2009). Pedestrian tracking using inertial sensors. Journal of Physical Agents, 3(1), 35-43.

Foxlin, E. (2005). Pedestrian tracking with shoe-mounted inertial sensors. IEEE Computer Graphics and Applications, 25(6), 38-46.

Godha, S. and Lachapelle, G. (2008). Foot mounted inertial system for pedestrian navigation. Measurement Science and Technology, 19(7), 075202.

Grejner-Brzezinska, D.A., Toth, C. K. and Yi, Y. (2001). Bridging GPS gaps in urban canyons: can ZUPT really help? Proceedings of the 58th Annual Meeting of the Institute of Navigation and CIGTF 21st Guidance Test Symposium, 231-240.

Han, S. and Wang, J. (2011). Quantization and coloured noises error modelling for inertial sensors for GPS/ INS integration. IEEE Sensors Journal, 11(6), 1493-1503.

Hide, C., Botterill, T. and Andreotti, M. (2010). Low cost vision-aided IMU for pedestrian navigation. In Ubiquitous Positioning Indoor Navigation and Location Based Service (UPINLBS), 1-7, Kirkkonummi, Finland.

Huang, C., Liao, Z. and Zhao, L. (2010). Synergism of INS and PDR in self-contained pedestrian tracking with a miniature sensor module. IEEE Sensors Journal, 10(8), 1349-1359.

Jiménez, A.R., Seco, F., Prieto, C. and Guevara, J. (2009). A comparison of pedestrian dead-reckoning algorithms using a low-cost MEMS IMU. IEEE International Symposium on Intelligent Signal Processing, 37-42, Budapest, Hungary.

Jiménez, A. R., Seco, F., Prieto, J.C. and Guevara, J. (2010). Indoor pedestrian navigation using an INS/ EKF framework for yaw drift reduction and a foot-mounted IMU. 7th Workshop on Positioning Navigation and Communication (WPNC), 135-143, Dresden, German.

Li, Y. and Wang, J.J. (2013). Zero velocity update with stepwise smoothing for inertial pedestrian navigation, IGNSS, Gold Coast, Queensland, Australia.

Li, Y., Wang, J.J., Xiao, S. and Luo, X. (2012). Dead reckoning navigation with constant velocity update (CUPT). Proceedings of the 12th International Conference on Control, Automation, Robotics and Vision (ICARCV2012), IEEE, 160-165, Guangzhou China.

Li, Y. and Wang, J.J. (2012). A robust pedestrian navigation algorithm with low cost IMU. International Conference on Indoor Positioning and Indoor Navigation (IPIN 2012), University of New South Wales, Sydney, Australia.

Ojeda, L. and Borenstein, J. (2007). Non-GPS navigation for security personnel and first responders. Journal of Navigation, 60(3), 391-407.

Park, S. K. and Suh, Y.S. (2010). A zero velocity detection algorithm using inertial sensors for pedestrian navigation systems. Sensors, 10(10), 9163-9178.

Rauch, H. E., Striebel, C T and Tung, F. (1965). Maximum likelihood estimates of linear dynamic systems. AIAA journal, 3(8), 1445-1450. 
Skog, I., Nilsson, J.O. and Handel, P.I. (2010). Evaluation of zero-velocity detectors for foot-mounted inertial navigation systems. 2010 International Conference on Indoor Positioning and Indoor Navigation (IPIN), Campus Science City, ETH Zurich.

Suh, Y.S. and Park, S. (2009). Pedestrian inertial navigation with gait phase detection assisted zero velocity updating. 4th International Conference on Autonomous Robots and Agents, Wellington, New Zealand.

Yun, X., Bachmann, E.R., Moore, H. and Calusdian, J. (2007). Self-contained position tracking of human movement using small inertial/magnetic sensor modules. 2007 IEEE International Conference on Robotics and Automation (ICRA), Roma, Italy.

Wang, J.J, Ding, W and Wang, J. (2007). Improving adaptive Kalman filter in GPS/SDINS integration with neural network. 20th Int. Tech. Meeting of the Satellite Division of the U.S. Inst. of Navigation, pp. 571-578, Fort Worth, Texas. 